\title{
Association between Dix-Hallpike test parameters and successful repositioning maneuver in posterior semicircular canal benign paroxysmal positional vertigo: a case-control study
}

\author{
Jia Yu", Guilin Meng", Shaofang Xu, Pengfei Chen, Xiaoqing Liu, Yanxin Zhao, Xueyuan Liu, Aiping Jin \\ Shanghai Tenth People's Hospital, Tongji University School of Medicine, Shanghai 200072, China \\ Contributions: (I) Conception and design: G Meng, X Liu; (II) Administrative support: Y Zhao; (III) Provision of study materials or patients: G Meng, \\ X Liu; (IV) Collection and assembly of data: S Xu, P Chen; (V) Data analysis and interpretation: G Meng, X Liu; (VI) Manuscript writing: All \\ authors; (VII) Final approval of manuscript: All authors. \\ \#These authors contributed equally to this work. \\ Correspondence to: Xueyuan Liu; Aiping Jin. Shanghai Tenth People's Hospital, Tongji University School of Medicine, Shanghai 200072, China. \\ Email: liuxy@tongji.edu.cn; 13402140058@163.com.
}

\begin{abstract}
Background: Nystagmus (i.e., involuntary eye movement) provoked by the Dix-Hallpike test (DHT) is considered the gold standard for diagnosing posterior semicircular canal benign paroxysmal positional vertigo (psc-BPPV). However, robust evidence regarding this diagnostic maneuver's treatment efficacy is still lacking. This study aimed to investigate the parameters of positional nystagmus in the DHT as prognostic factors for unilateral psc-BPPV.

Methods: We reviewed 357 patients with unilateral psc-BPPV who underwent the computer-controlled canalith repositioning procedure (CCRP), which mimics the Epley maneuver. Additionally, positional nystagmus in the DHT was observed through a video-oculography. Patients were retrospectively divided into two groups according to resistance to the CCRP. Univariable and multivariable analyses were performed on age, gender, blood pressure, and nystagmus parameters of the affected side in the DHT in order to reveal associated factors causing resistance to the CCRP.

Results: With univariate and multivariate analysis, we observed a decrease in vertical time course during slow phase nystagmus on the affected side (odds ratio, OR $0.77, \mathrm{P}=0.011$ vs. OR 0.80, $\mathrm{P}=0.027$ ). Moreover, an increased vertical velocity amplitude in the $\mathrm{DHT}$ during slow phase nystagmus (OR 3.16, $\mathrm{P}=0.029$ vs. OR 2.96, $\mathrm{P}=0.035$ ) remained an associated factor of maneuver resistance.

Conclusions: Decreased canalith weight on the affected side was associated with resistance to the CCRP. This association corresponded to the observation that psc-BPPV patients with a decreased time course and increased velocity in the slow phase of recording nystagmus have a worse prognosis of their BPPV persisting after a single CCRP.
\end{abstract}

Keywords: Benign paroxysmal positioning vertigo (BPPV); posterior semicircular canal; nystagmus; repositioning maneuvers

Submitted Oct 15, 2019. Accepted for publication Jan 30, 2020.

doi: $10.21037 /$ atm.2020.03.03

View this article at: http://dx.doi.org/10.21037/atm.2020.03.03

\section{Introduction}

Benign paroxysmal positioning vertigo (BPPV) is defined as a disorder of the inner ear characterized by repeated episodes of positional vertigo (1). BPPV accounts for nearly $30 \%$ of vertigo cases, with a lifetime prevalence of $2.4 \%$ in the general population $(2,3)$. The unilateral inner ear system contains three semicircular canals, including the anterior semicircular canal, horizontal semicircular 
canal, and posterior semicircular canal. Moreover, due to differences in the anatomy of the semicircular canal, the posterior semicircular canal happens to be the most frequently involved canal in BPPV (i.e., psc-BPPV), which accounts for $85-95 \%$ of BPPV cases (4). Despite the success of the canalith repositioning procedure (CRP) in treating psc-BPPV, there remains $20 \%$ of the BPPV population that does not benefit from CRP (5). Although BPPV is a benign vertigo disease and is somewhat self-limiting, the onset of vertigo, vertigo-associated symptoms, and residual symptoms in resistant BPPV affects a patient's health and quality-of-life. Furthermore, such symptomology may also leave patients, especially elderly patients, at a high-risk for catastrophic falls and a tremendous psychological burden (6).

Therefore, developing a test or parameter that could predict the efficacy of CRP would profoundly contribute to a more reasonably distributed patient care. There have been studies investigating how osteoporosis may be a key predictor of BPPV prognosis (7). Moreover, some studies have concluded that patients with osteoporosis have a higher risk of occurrence and recurrence of BPPV (8). However, other studies have shown a negative correlation between osteoporosis/vitamin D deficiency and BPPV (9). Other studies investigating the role of other prognostic factors, such as arteriosclerotic turn over (10), prior trauma or labyrinthitis (11), previous BPPV history, and intensity of nystagmus (12) predicted a low correlation for BPPV outcomes. Furthermore, abnormal vestibular evoked myogenic potentials (VEMPs), as neurophysiological parameters, showed a chronic and resistive BPPV prognosis (13), while vertigo occurring immediately after a maneuver as a liberator sign, correlated with the success of treatment (14). In BPPV patients with cervical vestibularevoked myogenic potential (cVEMP) or ocular VEMP (oVEMP) abnormalities, quality-of-life was reduced as compared to those without these abnormalities (15).

However, there is still little evidence concerning the immediate correlation between nystagmus parameters in the Dix-Hallpike test (DHT) with CRP efficacy; although, characteristic nystagmus provoked by DHT is essential for diagnosing psc-BPPV. Nevertheless, descriptions for characteristic upbeat nystagmus and torsional nystagmus (with the top pole of rotation beating toward the affected ear), such as specific parameters of velocity, time course, and angle of positional nystagmus remain inadequate. In the present study, we aimed to evaluate positional nystagmus parameters in the DHT as prognostic factors for psc-BPPV prognosis

\section{Methods}

\section{Study design}

This study was part of a retrospective analysis of BPPV efficacy approved by the Local Ethics Committee of Shanghai Tenth People's Hospital. Patients with pscBPPV in this case-control study were recruited from the vertigo neurology clinic in the hospital, one of the largest integrative vertigo clinics in Shanghai, between January 2017 and June 2019.

Patients were diagnosed with psc-BPPV according to clinical practice guidelines of the American Academy of Neurology (16). Briefly, the diagnostic criterion was based on patient-reported history of repeated episodes of vertigo with changes in head position and demonstrated characteristic vertigo and nystagmus provoked by the DHT.

The study invitation was sent when a patient visited the vertigo neurology clinic. If the patient agreed to attend the study, their electronic medical records would be reviewed for admission. After a detailed evaluation with inclusion and exclusion criteria, 357 patients were included in this study (Figure 1).

\section{Inclusion criteria}

Patients who met all of the following inclusion criteria were eligible for the study:

(I) Patients who adequately understood the purpose of the study agreed to participate and signed the informed consent files.

(II) Patients who were diagnosed with unilateral pscBPPV.

\section{Exclusion criteria}

Patients who met any of the following exclusion criteria were excluded from the study:

(I) Presence of eye diseases, or patients who kept their eyes closed where nystagmus could not be recorded.

(II) Presence of severe cardiovascular and/or cerebrovascular disease or multisystem functional failure.

(III) History of head trauma, sleep disorders, psychiatric disorders, or inner ear diseases.

(IV) Secondary or bilateral/multiple BPPV.

At admission, demographics, past medical history, and nystagmus data in the DHT and CRP were recorded. 


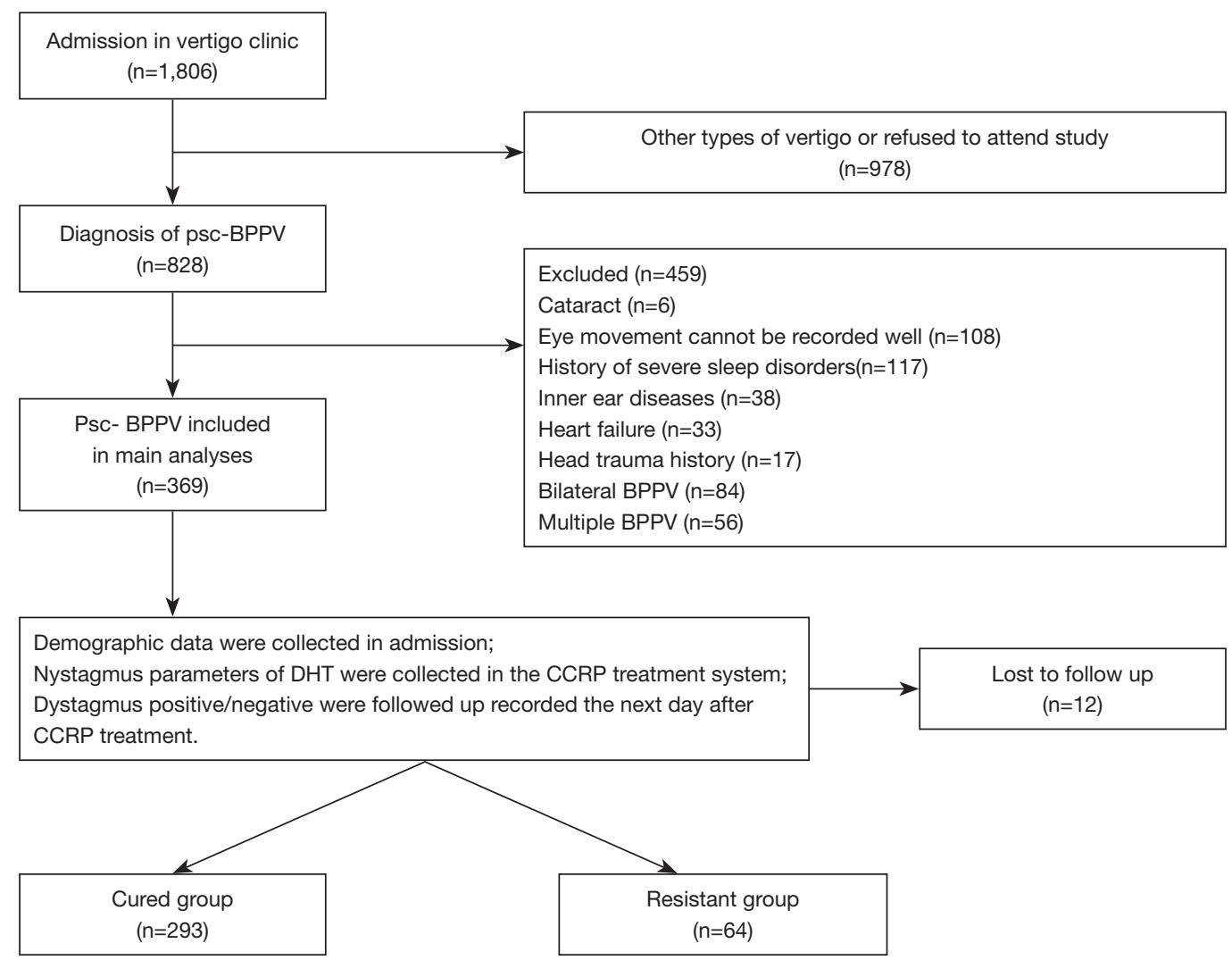

Figure 1 Flow chart of the study implementation. Psc-BPPV, posterior semicircular canal benign paroxysmal positional vertigo.

\section{Detailed DHT and CRP for psc-BPPV}

The DHT is the gold standard maneuver for diagnosing psc-BPPV, and the Epley technique is considered a classic method for psc-BPPV reset (16). The DHT and CRP were performed in a diagnosis and treatment system for BPPV (Byron's Medical Science \& Technique Inc., Jinan, China) as previously described (17). Using a computer-controlled CRP (CCRP), which still mimics Epley maneuver (EM) (18) parameters in the computer-setting instrument for the treatment of psc-BPPV, avoids the limitations of EM in some elderly patients with physical motor impairment or obesity.

The psc-BPPV was diagnosed by the DHT mode, in which upbeat and torsional nystagmus occurred less than $1 \mathrm{~min}$ when patients were placed in an automatic swivel chair with each action at $90 \%$ speed to complete. In right semicircular canal BPPV, for instance, the patient sits on the swivel chair, turns left $45^{\circ}$, then right-leaning back $120^{\circ}$. If nystagmus was induced in this position, it was observed and recorded. Then the patient was slowly returned to the starting position until nystagmus completely disappeared (Figure 2).

The treatment was carried out in the CCRP mode in this system. Each position shift in the fully automatic swivel chair was at $90^{\circ} \%$ s. For right semicircular canal BPPV reset, for example, once the induced nystagmus and vertigo completely disappeared in the DHT mode, the patient was continually rotated from a $120^{\circ}$ to $250^{\circ}$ position for $60 \mathrm{~s}$, then slowly rotated back to the starting position.

Positional nystagmus was observed through a videooculography where latency, direction, time course, and a max velocity were evaluated. Velocity is a vector that uses the orthogonal decomposition method to decompose the velocity into a horizontal $\mathrm{X}$-axis and a vertical Y-axis. The data in the CCRP process was much more complicated than in the DHT based on the procedure. Therefore, we at first aimed to analyze the DHT for maneuver efficacy; if the results were positive, we investigated the potential links in CCRP data in further studies (Figure 3). 

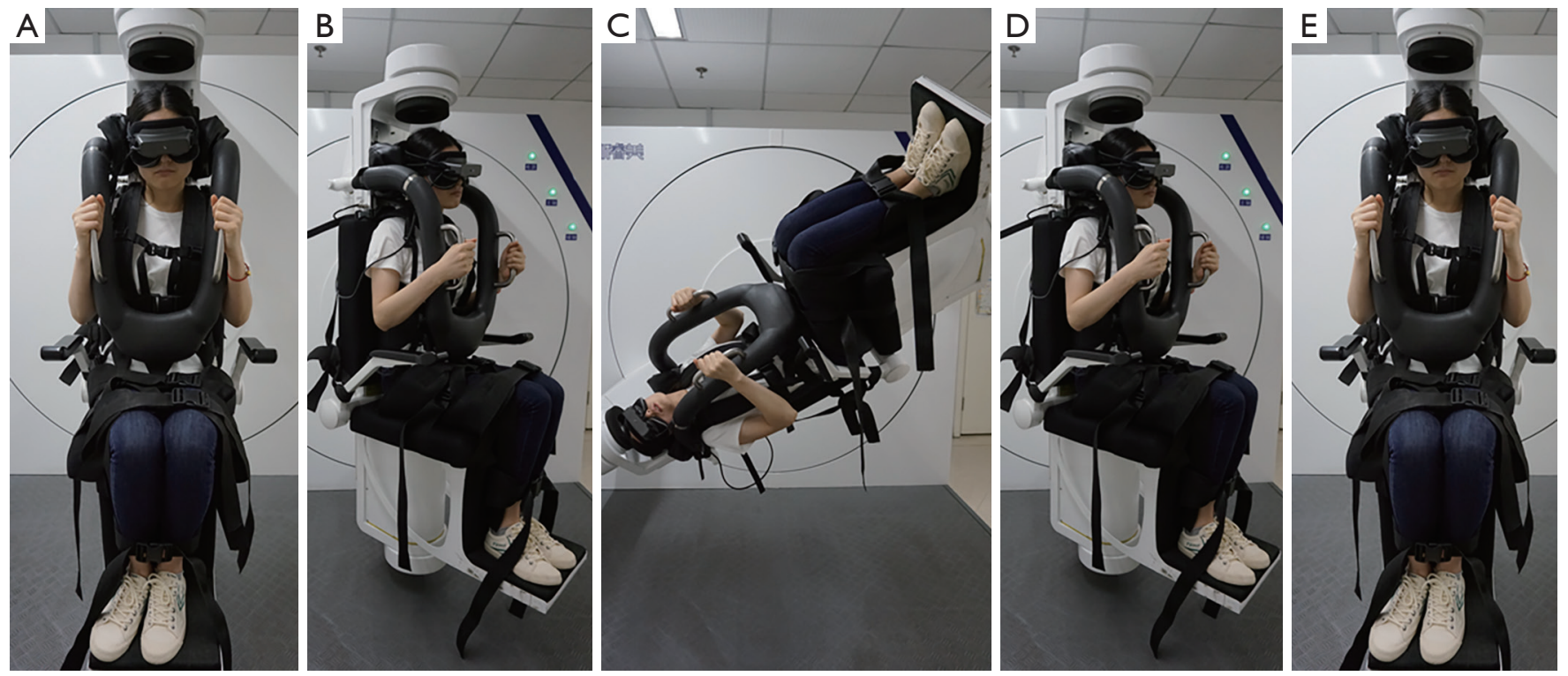

Figure 2 Dix-Hallpike test (DHT) mode for right semicircular canal BPPV. BPPV, benign paroxysmal positional vertigo.

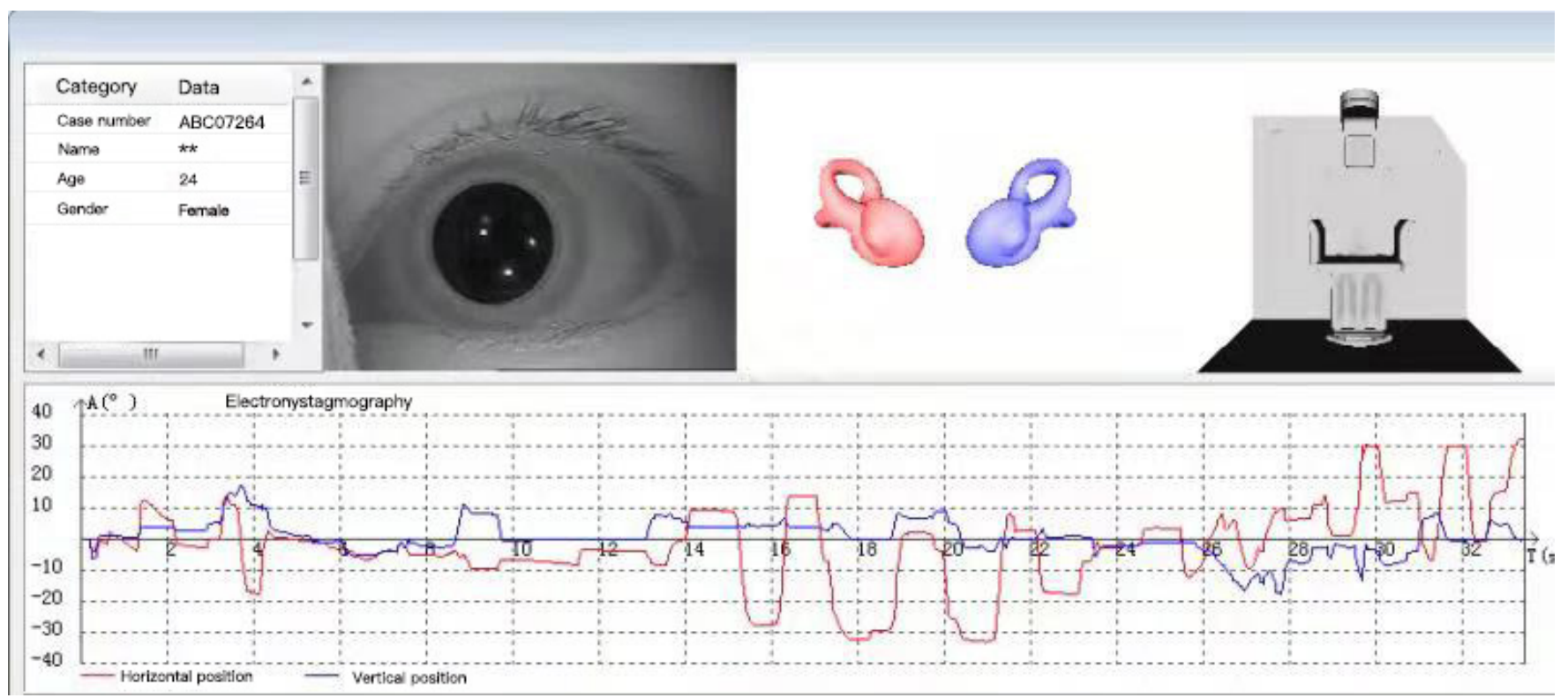

Figure 3 Nystagmus velocity in Dix-Hallpike test (DHT) decomposed into a horizontal X-axis and a vertical Y-axis (red as X, blue as Y).

\section{Primary outcome measurement and grouping}

Patients were followed up the day after the initial CCRP to assess the CCRP efficiency. A negative DHT, yielding no nystagmus at all, was considered a treatment success independent from patient-reported vertigo. This criterion was used as the primary outcome measure to evaluate the effectiveness of CCRP for the treatment of psc-BPPV (19). When provoked nystagmus on the DHT was absent, the patient was considered as having a resolution of pscBPPV. When a patient did not resolve, while exhibited positive nystagmus including without change, intensified, or turned to other types after the initial CCRP, the patient 
was considered to have resistant BPPV that would undergo further treatment with corresponding individual CRP based on the affected canal. Subsequent treatment results were not included in the analysis for the efficacy of initial CCRP in this study.

Patients who had successful treatment were classified as the "cured group", while those who did not resolve after initial CCRP were classified as the "resistant group".

\section{Measurement of potential confounders}

We measured foundational variables potentially that may affect the outcome. These potential confounders were demographic characteristics of age, gender, and blood pressure.

\section{Statistical analysis}

All analyses were carried out using SPSS 22.0 (SPSS Inc., Chicago, IL, USA). The Kolmogorov-Smirnov test was used to determine data distribution. Data variables were expressed as mean \pm standard deviation $(\mathrm{x} \pm \mathrm{SD})$ or $\mathrm{n}(\%)$ based on descriptive purposes. Independent sample $t$-test and Chi-square test were used to compare continuous and categorical variables, respectively. Univariable analysis was performed to identify factors with a potential significant association $(\mathrm{P}<0.05)$ with CCRP efficacy, following by multivariable logistic regression analysis modeling factor independent contributions. The OR [95\% confidence interval $(\mathrm{CI})$ ] were calculated for examining the association between risk factors of DHT parameters and treatment efficacy. All statistical assessments were two-tailed, and $\mathrm{P}<0.05$ was considered statistically significant.

\section{Results}

\section{General patient characteristics}

A total of 1,806 patients admitted to the vertigo clinic at the Shanghai Tenth People's Hospital affiliated to Tongji University between January 2017 and June 2019, in which 828 patients diagnosed with psc-BPPV were initially interviewed. In total, 459 patients were excluded due to a poor eye movement record (cataracts and inability to keep eyes opened for an extended period during the recording process), other medical history that affected vertigo performance (head trauma, inner ear diseases, and history of severe disorders), heart failure that made the patient not suitable for substantial repositioning movement, and/or bilateral/multiple BPPV. Twelve recording patients were lost for follow-up. In total, 357 patients qualified to fulfill the criteria for analysis, 293 were placed in the cured group, while the remaining 64 were placed in the resistant group. CCRP was performed without serious adverse effects in all patients. Side effects of CCRP were transient nausea, vomiting, and loss of balance, which occurred in 67 subjects. The average age $( \pm \mathrm{SD})$ of participants was $57.2 \pm 12.6$ years old (range, 27-79 years old), where $70.0 \%$ were female. In terms of comorbidities, female and affected left side canal were more prevalent in the resistant group $(\mathrm{P}=0.008$, and $\mathrm{P}=0.005$, respectively). In addition, diastolic blood pressure (DBP) was much lower in the resistant group than in the cured group $(\mathrm{P}=0.018)$. Systolic blood pressure (SBP) status and the hypertension rate were not significantly associated with CCRP efficacy (Table 1).

Table 2 shows comparisons of DHT parameters between the psc-BPPV cured group and resistant group. In the cured group, the mean slow phase time course on the Y-axis was almost 0.9 milliseconds (ms) longer than the resistant group $(\mathrm{P}=0.026)$, while no significant difference on the $\mathrm{X}$-axis slow phase time course was observed. According to the slow phase velocity, the amplitude on the Y-axis was nearly twice as much as on the $\mathrm{X}$-axis $(22.8 \pm 13.8 v s .11 .3 \pm 5.4)$, without differences in the two groups.

Univariate logistic regression identified the following demographic and DHT characteristics associated with CCRP resistance in psc-BPPV patients: age (OR 1.09; $\mathrm{P}=0.041$ ), male (OR 0.76; $\mathrm{P}=0.043$ ), slow phase velocity amplitude on the $\mathrm{Y}$-axis (OR 3.16, $\mathrm{P}=0.029)$, and slow phase time course on the $\mathrm{Y}$-axis (OR $0.77, \mathrm{P}=0.011$ ). In multivariable logistic regression, the gender influence disappeared, where only age (OR 1.05; $\mathrm{P}=0.047)$, slow phase velocity amplitude on the $\mathrm{Y}$-axis $(\mathrm{OR} 2.96, \mathrm{P}=0.035)$, and slow phase time course on the $\mathrm{Y}$-axis $(\mathrm{OR} 0.80, \mathrm{P}=0.027$ ) were associated characteristics (Table 3).

Besides the age influence, we confirmed the utility of the slow phase velocity and time course on the Y-axis of nystagmus parameters in the DHT as prognostic factors for the efficacy of CCRP treatment in most cases. Nevertheless, the faster the slow phase velocity, the shorter the time course on the Y-axis, and the worse the maneuver effect.

\section{Conclusions}

Compared with the conventional Epley maneuver, the 
Table 1 Baseline characteristics of patients with psc-BPPV cured or resistant after CCRP

\begin{tabular}{|c|c|c|c|c|}
\hline Characteristics & All patients $(n=357)$ & Cured group $(n=293)$ & Resistant group ( $n=64$ ) & $P$ value \\
\hline \multicolumn{5}{|l|}{ Gender (\%) } \\
\hline Male & $104(29.1)$ & $98(33.4)$ & $6(9.4)$ & \multirow[t]{2}{*}{$0.008^{\#}$} \\
\hline Female & $253(70.9)$ & $195(66.6)$ & $58(90.6)$ & \\
\hline \multicolumn{5}{|c|}{ Blood pressure (mmHg) } \\
\hline SBP & $130.1 \pm 13.7$ & $130.8 \pm 13.4$ & $126.7 \pm 14.5$ & 0.299 \\
\hline DBP & $75.6 \pm 10.2$ & $76.5 \pm 10.4$ & $70.9 \pm 8.1$ & $0.018^{*}$ \\
\hline \multicolumn{5}{|l|}{ Affected side (\%) } \\
\hline
\end{tabular}

$\mathrm{P}$ values comparing persons in cured group and resistant group. *, $\mathrm{P}<0.05$ vs. resistant group; ${ }^{\#}, \mathrm{P}<0.01$ vs. resistant group. psc-BPPV, posterior semicircular canal benign paroxysmal positional vertigo; CCRP, computer-controlled canalith repositioning procedure; SBP, systolic blood pressure; DBP, diastolic blood pressure.

Table 2 Comparison of DHT parameters between patients with psc-BPPV cured or resistant after CCRP

\begin{tabular}{lccc}
\hline Characteristics & All patients $(\mathrm{n}=357)$ & Cured group $(\mathrm{n}=293)$ & Resistant group $(\mathrm{n}=64)$ \\
\hline SP velocity & & & $\mathrm{P}$ value \\
$\mathrm{X}$ amplitude $(\mu \mathrm{m} / \mathrm{s})$ & $11.3 \pm 5.4$ & $11.5 \pm 5.8$ & $10.7 \pm 3.8$ \\
Y amplitude $(\mu \mathrm{m} / \mathrm{s})$ & $22.8 \pm 13.8$ & $23.4 \pm 13.8$ & $21.0 \pm 13.6$ \\
SP time course & & & 0.615 \\
X $(\mathrm{ms})$ & $4.2 \pm 2.2$ & $4.5 \pm 2.7$ & $3.9 \pm 1.9$ \\
$\mathrm{Y}(\mathrm{ms})$ & $5.5 \pm 3.3$ & $5.7 \pm 3.4$ & $4.8 \pm 3.0$ \\
\hline
\end{tabular}

$\mathrm{P}$ values comparing persons in cured group and resistant group. ${ }^{*}, \mathrm{P}<0.05$ vs. resistant group. psc-BPPV, posterior semicircular canal benign paroxysmal positional vertigo; DHT, Dix-Hallpike test; CCRP, computer-controlled canalith repositioning procedure; SP, slow phase; $\mathrm{SD}$, standard deviation.

modified computational system can be quantitative to fix speed and position, overcoming manual reset shortcomings of personal experience. As mentioned above, approximately $18 \%$ of patients treated after CCRP complained about experiencing dizziness, balance disorders, floating sense and instability, and other atypical complaints to the treatment, which is less common than in the routine Epley maneuver (20). Given the use of three-dimensional animation and high-definition display technology, the system of nystagmus and the recording of the nystagmus curve are more sophisticated, providing favorable conditions for accurate resetting. Efficient therapy can effectively shorten the course of the disease; reduce patients' pain, thereby improving the quality-of-life of patients; reduce the risk of falls; and avoid several unnecessary or invalid examinations and treatments.

Although the qualitative nystagmus comparison of different outcomes of BPPV has been previously described, such as the Epley maneuver being an effective method to reverse a positive DHT and reduced vertigo severity in patients with DHT-based linear nystagmus (21); reported nystagmus in the same direction at the second position of the Epley maneuver as noted on the DHT is associated with therapeutic success (22). The reversed nystagmus was correlated with a CRP failure (23), and the type of nystagmus observed at the Semont maneuver has been 
Table 3 Univariate and multivariable analyses of demographic and DHT predictors of CCRP resistance in psc-BPPV patients

\begin{tabular}{|c|c|c|c|c|}
\hline Variables & \multicolumn{2}{|c|}{ Univariate } & \multicolumn{2}{|c|}{ Multivariate } \\
\hline Age, years & $1.09(1.08-1.11)$ & $0.041^{*}$ & 1.05 (1.04-1.07) & $0.047^{*}$ \\
\hline Male & $0.76(0.70-0.82)$ & $0.043^{*}$ & $0.89(0.72-1.03)$ & 0.224 \\
\hline Hypertension & $1.31(0.89-1.55)$ & 0.442 & $1.02(0.88-1.12)$ & 0.401 \\
\hline \multicolumn{5}{|l|}{ DHT } \\
\hline \multicolumn{5}{|l|}{ SP velocity } \\
\hline X amplitude & $0.84(0.69-1.11)$ & 0.554 & $0.89(0.93-1.05)$ & 0.619 \\
\hline Y amplitude & $3.16(2.04-4.19)$ & $0.029^{*}$ & $2.96(2.21-3.97)$ & $0.035^{\star}$ \\
\hline$Y$ & $0.77(0.59-0.82)$ & $0.011^{*}$ & $0.80(0.74-0.88)$ & $0.027^{\star}$ \\
\hline
\end{tabular}

*, $\mathrm{P}<0.05$. Cl, confidence interval; OR, odds ratio; $\mathrm{DHT}$, Dix-Hallpike test; CCRP, computer-controlled canalith repositioning procedure; psc-BPPV, posterior semicircular canal benign paroxysmal positional vertigo; SP, slow phase.

proposed as useful adjuncts in predicting outcomes (24). Nevertheless, the analysis of quantitative parameters has been exhaustively studied and remains controversial. Domínguez-Durán et al. suggested a tendency towards poorer maneuver's prognosis with higher vertigo intensity in the Dix-Hallpike maneuver. In the second phase of the $\mathrm{EM}$, orthotropic nystagmus indicates a better prognosis, but inverted orthotropic nystagmus indicates a poorer prognosis (12). Yetişer et al. compared the nystagmus characteristics of slow-phase velocity and duration between lateral canal and posterior canal BPPV. They demonstrated that it was not statistically significant (25), while Kim et al. proposed that nystagmus characteristics during the CRP as an objective predictive parameter for maneuver success (26). We found that resistant psc-BPPV patients often have typical induced nystagmus in the vertical direction, with faster velocity and a shorter time duration during the slow phase. Identification of such patients through detailed observation and precise diagnosis provides a good condition for clinical management.

Several patterns of velocity and duration can be explained by the time the canalith takes to reach the duct, various sizes of the canalith, and wall-particle interactions. In a mathematical BPPV model, a large quantity of small canalith particles caused more severe nystagmus than fewer bigger particles (27). The latency of slow phase nystagmus is explained by the delay in setting the canalith debris into the primary semicircular canal position. The movement of the canalith is decomposed, i.e., horizontally and vertically. The faster the velocity amplitude, the shorter peak time duration, which means the greater the acceleration of the otolith in this direction. The acceleration in the horizontal direction is related to the rolling friction coefficient $(\mu)$ during otolith movement. This value is fixed in the same material; thus, there is almost no difference in horizontal nystagmus. The acceleration in the vertical direction is inversely proportional to the mass of the canalith, meaning that the smaller the mass of the canalith, the greater the acceleration in the vertical direction. This can also be further understood by observing that vertigo, caused by finely divided canalith particles, is more difficult to reset in accordance with previous study results (27). Incomplete improvement and perseverance of symptoms may also occur, and it may be attributed to the presence of anatomical variations in the canal diameter and length. Additionally, more extensive studies with longer follow-ups as well as other types of BPPV should be conducted in the future.

Unilateral psc-BPPV patients with increased vertical velocity and decreased vertical time course in slow-phase nystagmus parameters in the DHT at the affected side have a worse prognosis of their BPPV persisting after a single CCRP. These results might be useful for clinicians in recognizing and treating psc-BPPV. 


\section{Acknowledgments}

The authors thank the patients for their commitment to this research.

Funding: This study was supported by the Excellent Young Physician Project of Shanghai Tenth People's Hospital (No. 2018SYPDRC038), Shanghai Sailing Program (No. 19YF1438400), and the Shanghai Science and Technology Commission Project (No. 18411961700, 17411950100, and 19441908500).

\section{Footnote}

Conflicts of Interest: The authors have no conflicts of interest to declare.

Ethical Statement: The authors are accountable for all aspects of the work in ensuring that questions related to the accuracy or integrity of any part of the work are appropriately investigated and resolved. Committee of Shanghai Tenth People's Hospital approved the study (ethical approval number: SHSY-IEC-4.0/18-44/01). All participants signed informed consent.

Open Access Statement: This is an Open Access article distributed in accordance with the Creative Commons Attribution-NonCommercial-NoDerivs 4.0 International License (CC BY-NC-ND 4.0), which permits the noncommercial replication and distribution of the article with the strict proviso that no changes or edits are made and the original work is properly cited (including links to both the formal publication through the relevant DOI and the license). See: https://creativecommons.org/licenses/by-nc-nd/4.0/.

\section{References}

1. Bhattacharyya N, Gubbels SP, Schwartz SR, et al. Clinical Practice Guideline: Benign Paroxysmal Positional Vertigo (Update). Otolaryngol Head Neck Surg 2017;156:S1-47.

2. Balatsouras DG, Koukoutsis G, Fassolis Benign A, et al. Paroxysmal Positional Vertigo in the Elderly: Current Insights. Clin Interv Aging 2018;13:2251-66.

3. von Brevern M, Radtke A, Lezius F, et al. Epidemiology of benign paroxysmal positional vertigo: a population based study. J Neurol Neurosurg Psychiatry 2007;78:710-5.

4. Bhattacharyya N, Baugh RF, Orvidas L, et al. Clinical practice guideline: benign paroxysmal positional vertigo. Otolaryngol Head Neck Surg 2008;139:S47-81.
5. Gaur S, Awasthi SK, Bhadouriya SK, et al. Efficacy of Epley's Maneuver in Treating BPPV Patients: A Prospective Observational Study. Int J Otolaryngol 2015;2015:487160.

6. You P, Instrum R, Parnes L. Benign paroxysmal positional vertigo. Laryngoscope Investig Otolaryngol 2018;4:116-23.

7. Yu S, Liu F, Cheng Z, et al. Association between osteoporosis and benign paroxysmal positional vertigo: a systematic review. BMC Neurol 2014;14:110.

8. Byun H, Chung JH, Lee SH, et al. Increased Risk of Benign Paroxysmal Positional Vertigo in Osteoporosis: A Nationwide Population-Based Cohort Study. Sci Rep 2019;9:3469.

9. Karataş A, Acar Yuceant G, Yuce T, et al. Association of Benign Paroxysmal Positional Vertigo with Osteoporosis and Vitamin D Deficiency: A Case Controlled Study. J Int Adv Otol 2017;13:259-65.

10. Wada M, Naganuma H, Tokumasu K, et al. Correlation between arteriosclerotic changes and prognosis in patients with peripheral vestibular disorders. Int Tinnitus J 2009;15:193-5.

11. Del Rio M, Arriaga MA. Benign positional vertigo: prognostic factors. Otolaryngol Head Neck Surg 2004;130:426-9.

12. Domínguez-Durán E, Domènech-Vadillo E, ÁlvarezMorujo de Sande MG, et al. Analysis of risk factors influencing the outcome of the Epley maneuver. Eur Arch Otorhinolaryngol 2017;274:3567-76.

13. Sreenivasan A, Sivaraman G, Parida PK, et al. The Clinical Utility of Vestibular Evoked Myogenic Potentials in Patients of Benign Paroxysmal Positional Vertigo. J Clin Diagn Res 2015;9:MC01-03.

14. Tirelli G, Boscolo Nata F, Gardenal N, et al. Liberatory vertigo: a new prognostic factor for repositioning maneuvers. Am J Emerg Med 2016;34:1548-51.

15. Hoseinabadi R, Pourbakht A, Yazdani N, et al. The effects of abnormality of cVEMP and oVEMP on rehabilitation outcomes in patients with idiopathic benign paroxysmal positional vertigo. Eur Arch Otorhinolaryngol 2016;273:643-8.

16. Fife TD, Iverson DJ, Lempert T, et al. Quality Standards Subcommittee AAoN Practice parameter: therapies for benign paroxysmal positional vertigo (an evidence-based review): report of the Quality Standards Subcommittee of the American Academy of Neurology. Neurology 2008;70:2067-74.

17. Shan X, Peng X, Wang E. Efficacy of computer-controlled repositioning procedure for benign paroxysmal positional 
vertigo. Laryngoscope 2015;125:715-9.

18. Song MH, Kong TH, Shim DB. Optimal reassessment time for treatment response in posterior canal benign paroxysmal positional vertigo. Laryngoscope 2020;130:496-9.

19. Chang MY, Shin JH, Oh KH, et al. Clinical implication of cervical vestibular evoked myogenic potentials in benign paroxysmal positional vertigo. Clin Neurophysiol 2017;128:351-6.

20. Power L, Murray K, Bullus K, et al. Central Conditions Mimicking Benign Paroxysmal Positional Vertigo: A Case Series. J Neurol Phys Ther 2019;43:186-91.

21. Ballvé JL, Carrillo-Muñoz R, Rando-Matos Y, et al. Effectiveness of the Epley Manoeuvre in Posterior Canal Benign Paroxysmal Positional Vertigo: A Randomised Clinical Trial in Primary Care. Br J Gen Pract 2019;69:e52-60.

22. Fyrmpas G, Barkoulas E, Haidich AB, et al. Vertigo during the Epley maneuver and success rate in patients with BPPV. Eur Arch Otorhinolaryngol 2013;270:2621-5.

23. Oh HJ, Kim JS, Han BI, et al. Predicting a successful treatment in posterior canal benign paroxysmal positional vertigo. Neurology 2007;68:1219-22.

24. Soto-Varela A, Rossi-Izquierdo M, Santos-Perez S. Can we predict the efficacy of the semont maneuver in the treatment of benign paroxysmal positional vertigo of the posterior semicircular canal? Otol Neurotol 2011;32:1008-11.

25. Yetişer S, Ince D. Caloric Analysis of Patients with Benign Paroxysmal Positional Vertigo. J Int Adv Otol 2017;13:390-3.

26. Kim JS, Zee DS. Clinical practice. Benign paroxysmal positional vertigo. N Engl J Med 2014;370:1138-47.

27. Hain TC, Squires TM, Stone HA. Clinical implications of a mathematical model of benign paroxysmal positional vertigo. Ann N Y Acad Sci 2005;1039:384-94.

Cite this article as: Yu J, Meng G, Xu S, Chen P, Liu X, Zhao Y, Liu X, Jin A. Association between Dix-Hallpike test parameters and successful repositioning maneuver in posterior semicircular canal benign paroxysmal positional vertigo: a case-control study. Ann Transl Med 2020;8(6):286. doi: 10.21037/atm.2020.03.03 